\title{
Noble-metal nanocluster as enzyme-mimetic catalyst for diagnostic analysis
}

\author{
REN XiaoJun, WANG ShiZheng, SU DongDong, GAO Liang, YUAN Qing \& GAO XueYun*
}

Department of Chemistry and Chemical Engineering, Beijing University of Technology, Beijing 100124, China

Received August 20, 2019; accepted October 8, 2019; published online October 24, 2019

$\begin{array}{ll}\text { Citation: } & \text { Ren X J, Wang S Z, Su D D, et al. Noble-metal nanocluster as enzyme-mimetic catalyst for diagnostic analysis. Sci China Tech Sci, 2019, 62: 2306- } \\ & \text { 2309, https://doi.org/10.1007/s11431-019-1459-3 }\end{array}$

Noble-metal nanoclusters, which consist of only several to a few hundred metal atoms, have attracted a great deal of scientific interest due to their fascinating optical properties, biocompatibility and low toxicity [1]. Intriguingly, nanoclusters have been recently reported possessing intrinsic enzyme-mimetic properties, mainly including peroxidaselike activity, which can convert hydrogen peroxide into hydroxyl radicals, and catalase-like activity, which can convert hydrogen peroxide into water and oxygen. As low-cost alternatives to natural enzymes, nanoclusters can overcome several drawbacks of natural enzymes, such as sensitivity of catalytic activity to environmental conditions, low stability leaded by denaturation and digestion and high cost. In comparison to other nanomaterial based enzyme mimetics, nanoclusters are more prominent for bioanalysis due to their ultrasmall size, accurate molecular formula and excellent stability [2]. Diagnosis applications have recently started to emerge for nanoclusters with enzyme-mimetic properties, including biomarker detection, bio-imaging, protection against neurodegeneration and tumor prevention (Figure 1) [3].

Bovine serum albumin (BSA) stabilized Au nanoclusters (Au NCs) have firstly been found to possess highly intrinsic peroxidase-like activity, which can convert hydrogen peroxide into hydroxyl radicals. Consequently, most of reports for analyte detection are based on hydrogen peroxide detection by combining peroxidase mimicking nanoclusters

*Corresponding author (email: gaoxy@ihep.ac.cn) with a reporter substrate such as 3,3,5,5-tetramethylbenzidine (TMB) or luminol. This process can achieve the naked eye readout, which offers outstanding advantages of low cost, simple operation process, lack of requirement for complicated equipment, and easy-to-use applications. This colorimetric method can meet the need of on-site and read time detection, therefore hold potential applications for colorimetric sensors in biological and diagnosis areas. Based on the BSA-Au NCs as peroxidase mimetics, Wang et al. [4] developed a colorimetric method for xanthine detection. By combining intrinsic peroxidaselike activity and fluorescence properties of BSA-Au NCs, Tao et al. [5] developed a dual colorimetric and fluorometric sensor for highly sensitive and selective detection of dopamine in real samples. In addition, other noble-metal nanoclusters have also been found to possess intrinsic peroxidase-like activity, such as BSA stabilized copper nanoclusters $(\mathrm{Cu} \mathrm{NCs})$ [6], yeast extract stabilized Pt nanoclusters ( $\mathrm{Pt} \mathrm{NCs}$ ) [7], monothiolated $\beta$-cyclodextrin stabilized $\mathrm{Cu}$ NCs [8], DNA-templated $\mathrm{Ag} / \mathrm{Pt}$ bimetallic nanoclusters (DNA-Ag/Pt NCs) [9] and so on. Some have been developed as highly sensitive colorimetric sensors for glucose or thrombin detection. Recently, amino acid stabilized AuNCs have been reported to have similar peroxidasemimic activity like protein stabilized AuNCs. Amino acids are remarkable for their ambidentate nature and capablility of forming stable complexes with various metal ions. Based on histidine's ambidentate character, Liu et al. [10] rationally designed histidine stabilized AuNCs for ultrasensitive col- 


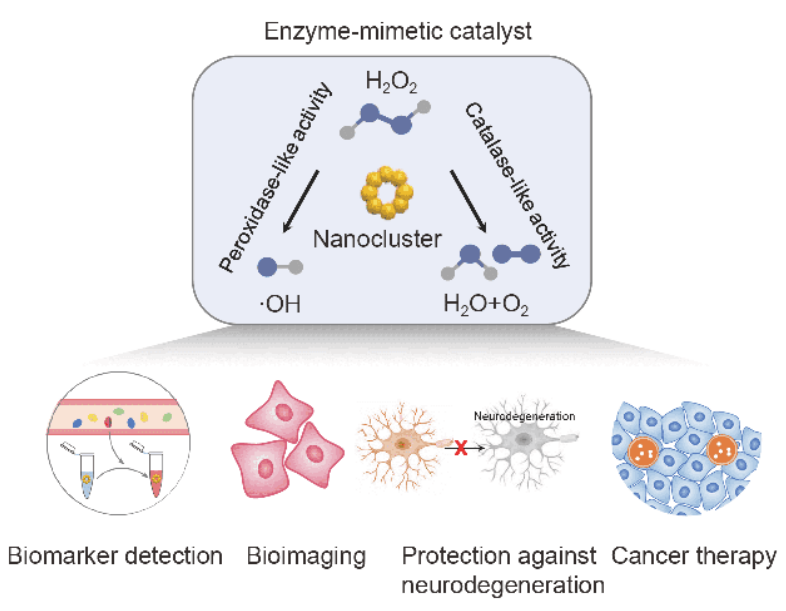

Figure 1 (Color online) Noble-metal nanocluster as enzyme-mimetic catalyst for diagnostic analysis.

orimetric assay of copper ions $\left(\mathrm{Cu}^{2+}\right)$ and histidine. The peroxidase-like activity of His-AuNCs can be efficiently inhibited in the presence of $\mathrm{Cu}^{2+}$. Then upon addition of histidine into His-AuNCs $/ \mathrm{Cu}^{2+}$ system, the chelation between the imidazole group of histidine and $\mathrm{Cu}^{2+}$ leads to $\mathrm{Cu}^{2+}$ release from His-AuNCs, and results in a significant recovery of enzyme activity of His-AuNCs.

Subsequently, nanoclusters have been applied for immunoassays such as ELISAs or capture-detection assays via conjugating antibodies or other targeting ligands. For example, based on a sandwiched antibody-antigen (or other analytes)-antibody structure, Zhao et al. [11] conjugated enzyme-mimetic $\mathrm{Au} \mathrm{NCs}$ on the outer layer antibody to catalyze hydrogen peroxide decomposition which was used to reduce $\mathrm{HAuCl}_{4}$ into gold nanoparticles for naked eye readout. This plasmonic nanosensor demonstrated high applicability for a variety of ultratrace analytes such as protein avidin, thyroid hormone, cancer antigen, and methamphetamine (MA), reaching ultrahigh sensitivity, for instant, nearly single molecular detection of MA.

Owing to their small size which is one or more orders of magnitude smaller than cells, nanoclusters can be taken up by cells or penetrate into tissues by passing between cells and diffusing through the extracellular matrix. Thus nanoclusters exhibit a clinical potential for bioimaging, cancer diagnosis and therapy $[12,13]$. Since folate receptors are underexpressed in normal tissues and overexpressed on the surface of different types of cancer cells, Hu et al. [14] developed a novel fluorescence enzyme mimetic nanoprobe based on folate receptor-targeting AuNCs. The nanoprobe could achieve peroxidase staining and fluorescent staining of the same tumor tissue slice simultaneously, and the results were mutually complementary. Nevertheless, the potential of AuNCs as enzyme mimic has also been limited by the low catalytic activity at neutral $\mathrm{pH}$. Remarkably, mental nanoclusters can be flexibly engineered to modify their prop- erties and boost catalytic activity. Currently, it is reported that graphene oxide (GO) could serve as the enzyme modulator to regulate the peroxidase-like activity of lysozyme stabilized AuNCs [15]. GO-AuNCs hybrid exhibits high catalytic activity over a broad $\mathrm{pH}$ range, even at neutral $\mathrm{pH}$. As GO possesses high surface-to-volume ratios and high affinity for hydrophobic molecules, it could bind to substrate and catalyst in the same nanoscale region, which could greatly enhance the catalytic activity of AuNCs. Qu's group conjugated folic acid on GO-AuNCs hybrid and developed a fast, cheap, and selective colorimetric approach to detect cancer cells at physical $\mathrm{pH}$. Inspired by these favorable results, our group has also synthesized peptide stabilized AuNCs for cancer cell detection [16]. The peptide can be designed to specifically target Membrane type 1 matrix metalloproteinase (MT1-MMP) which is positive regulators of tumor development, invasion and metastasis. By utilizing the intrinsic peroxidase-like activity of the AuNC probes, we can achieve assessing the expression level of MT1-MMP on the SH-SY5Y cells by the naked eye without cell lysis and protein extraction process. Meanwhile, Au count determined by inductively coupled plasma mass spectrometry (ICP-MS) and fluorescence intensity were acquired to verify the feasibility of this method. More importantly, due to the accurate molecular formula of peptide-cluster probe, we combined laser ablation inductively coupled plasma mass spectrometry (LA-ICP-MS) technique for single cell Au count determination, thus achieved precise quantification of membrane proteins expression level on single cells [17].

The emergence of the enzyme-mimetic properties of these nanoclusters has created new and exciting therapeutic opportunities beyond the traditional biomedical applications as carriers or for their optical properties. Recent works have demonstrated that mental nanoclusters can maintain catalytic processes under physiological conditions and provide therapeutic activity. Our group has explored peptide-coated $\mathrm{Au}$ NCs to selectively enhance the tumor-specific oxidative pressure with high efficiency [18]. By rationally designing peptide sequences to target integrin $\alpha_{v} \beta_{3}$ which was overexpressed on glioblastoma cells, the peptide-coated AuNCs can selectively recognize integrin on tumor cells and enter the cells. Then it catalyzes $\mathrm{H}_{2} \mathrm{O}_{2}$ to produce higher-toxic superoxide anion radicals $\left(\mathrm{O}^{2-}\right)$ and leads to the obvious enhancement of the reactive oxygen species (ROS) level in tumor cells, which thus induce mitochondria-dependent apoptosis and achieve high efficient anticancer thrapy [19]. Lin and their colleagues [20] have reported for the first time that amine-terminated PAMAM dendrimer-entrapped gold nanoclusters (AuNCs- $\mathrm{NH}_{2}$ ) lose their peroxidase-like activity unexpectedly while still retaining catalase-like activity in physiological conditions. The reason was proposed that the enrichment of polymeric $3^{\circ}$-amines on the surface of AuNCs- $\mathrm{NH}_{2}$ significantly suppressed the pivotal mediator 
- $\mathrm{OH}$ for the peroxidase-like activity. Due to the inhibition of intrinsic peroxidase-like activity, AuNCs- $\mathrm{NH}_{2}$ was conferred with diminished cytotoxicity, which therefore show huge potential for safe and long-term use. As ROS are the fundamental mediators of neurotoxicity in ischemia or reperfusion injury [21], the remained catalase-like activity of AuNCs- $\mathrm{NH}_{2}$ was used for neuroprotection without enzyme encapsulation and controllable release. It is indicated that AuNCs- $\mathrm{NH}_{2}$ treatment show an efficient suppression of intracellular $\mathrm{H}_{2} \mathrm{O}_{2}$, which protecting neurons against $\mathrm{H}_{2} \mathrm{O}_{2}-$ induced toxicity. By making use of this exciting feature, their group further employed AuNCs- $\mathrm{NH}_{2}$ for photodynamic therapy [22] (PDT) against hypoxia of cancer cells. AuNCs$\mathrm{NH}_{2}$ can self-supply $\mathrm{O}_{2}$ for PDT because that their intrinsic catalase-like activity can catalyze physiological $\mathrm{H}_{2} \mathrm{O}_{2}$ to generate $\mathrm{O}_{2}$. Importantly, attributed to their surface of dendrimers, AuNCs- $\mathrm{NH}_{2}$ extended the catalase-like activity to acidic conditions. They found that sequential treatments of hypoxic cancer cells with AuNCs- $\mathrm{NH}_{2}$ and photosensitizers before PDT significantly enhanced the PDT efficacy [23]. This study holds great promise for potential applications such as PDT to achieve more effective anticancer treatments.

Despite exciting progress in the development of metal nanoclusters with enzyme-mimetic properties in diagnostic applications, there are still considerable hurdles and challenges remaining with respect to promoting the performance of metal nanoclusters. Firstly, nanoclusters usually exhibit relatively low catalytic activity compared with natural enzymes. Changing the functional groups in the catalytic site or designing nanomaterials cores will be promising means to obtain high catalytic performance. Alternatively, exploring efficient modulators can also tailor the catalytic activities of these metal nanoclusters. Another important issue is that most of the work using catalytic nanoclusters has been performed prevailingly in vitro without taking account of the complexity of biological systems. Enzymatic properties and kinetics of nanoclusters can be affected by their microenvironment [24] and are challenging to control. The surface chemistries of nanoclusters can also be changed in the presence of biological fluids. It will be more valuable to investigate factors which affect catalytic activity and long-term effects of nanoclusters in biological systems. For example, developing new biomolecule-templated metal nanoclusters or increasing the surface-to volume ratio and the population of low-coordination sites, will be favorable for enhancing in vivo compatibility and broadening theranostic applications. In addition, these nanoclusters are limited to catalyze only two types of reactions, peroxidase-like and catalase-like reactions, whereas natural enzymes are known to catalyze a wide range of important biochemical reactions. Therefore, exploring the potential of nanoclusters for catalyzing new types of reactions are indeed essential. The field of nanoclusters enzyme mimetics is still in its infancy. Future directions should be centered on achieving maximal efficacy, stability and specificity with long-term effects and minimal toxicity as well as developing cost-effective formulations, which would be critical for application in clinical diagnostics.

1 Tao Y, Li M, Ren J, et al. Metal nanoclusters: novel probes for diagnostic and therapeutic applications. Chem Soc Rev, 2015, 44: 86368663

2 Cormode D P, Gao L, Koo H. Emerging biomedical applications of enzyme-like catalytic nanomaterials. Trends Biotech, 2018, 36: 15-29

3 Chen S, Liang X J. Nanobiotechnology and nanomedicine: Small change brings big difference. Sci China Life Sci, 2018, 61: 371-372

4 Wang X X, Wu Q, Shan Z, et al. BSA-stabilized Au clusters as peroxidase mimetics for use in xanthine detection. Biosens Bioelectron, 2011, 26: 3614-3619

5 Tao Y, Lin Y, Ren J, et al. A dual fluorometric and colorimetric sensor for dopamine based on BSA-stabilized Aunanoclusters. Biosens Bioelectron, 2013, 42: 41-46

$6 \mathrm{Hu} \mathrm{L}$, Yuan Y, Zhang L, et al. Copper nanoclusters as peroxidase mimetics and their applications to $\mathrm{H}_{2} \mathrm{O}_{2}$ and glucose detection. Anal Chim Acta, 2013, 762: 83-86

7 Jin L, Meng Z, Zhang Y, et al. Ultrasmall Pt nanoclusters as robust peroxidase mimics for colorimetric detection of glucose in human serum. ACS Appl Mater Interf, 2017, 9: 10027-10033

8 Zhong Y, Deng C, He Y, et al. Exploring a monothiolated $\beta$-cyclodextrin as the template to synthesize copper nanoclusters with exceptionally increased peroxidase-like activity. Microchim Acta, 2016, 183: $2823-2830$

9 Zheng C, Zheng A X, Liu B, et al. One-pot synthesized DNA-templated $\mathrm{Ag} / \mathrm{Pt}$ bimetallic nanoclusters as peroxidase mimics for colorimetric detection of thrombin. Chem Commun, 2014, 50: 1310313106

10 Liu Y, Ding D, Zhen Y, et al. Amino acid-mediated "turn-off/turn-on" nanozyme activity of gold nanoclusters for sensitive and selective detection of copper ions and histidine. Biosens Bioelectron, 2017, 92 : 140-146

11 Zhao Q, Huang H, Zhang L, et al. Strategy to fabricate naked-eye readout ultrasensitive plasmonic nanosensor based on enzyme mimetic gold nanoclusters. Anal Chem, 2015, 88: 1412-1418

12 Zhang H, Liu X L, Zhang Y F, et al. Magnetic nanoparticles based cancer therapy: Current status and applications. Sci China Life Sci, 2018, 61: 400-414

13 Ren X, Zhang K, Deng R, et al. RNA splicing analysis: from in vitro testing to single-cell imaging. Chem, 2019, 5: 2571-2592

$14 \mathrm{Hu} \mathrm{D}$, Sheng Z, Fang S, et al. Folate receptor-targeting gold nanoclusters as fluorescence enzyme mimetic nanoprobes for tumor molecular colocalization diagnosis. Theranostics, 2014, 4: 142-153

15 Tao Y, Lin Y, Huang Z, et al. Incorporating graphene oxide and gold nanoclusters: A synergistic catalyst with surprisingly high peroxidaselike activity over a broad $\mathrm{pH}$ range and its application for cancer cell detection. Adv Mater, 2013, 25: 2594-2599

16 Zhang X, Yuan Q, Gao X. Assessment of the MT1-MMP expression level of different cell lines by the naked eye. Sci China Life Sci, 2018, 61: 492-500

17 Gao L, Liu M, Ma G, et al. Peptide-conjugated gold nanoprobe: intrinsic nanozyme-linked immunsorbant assay of integrin expression level on cell membrane. ACS Nano, 2015, 9: 10979-10990

18 Zhang Y, Zhang X, Yuan Q, et al. Peptide-templated gold clusters as enzyme-like catalyst boost intracellular oxidative pressure and induce tumor-specific cell apoptosis. Nanomaterials, 2018, 8: 1040

19 Jin Y, Chen S, Li N, et al. Defect-related luminescent bur-like hydroxyapatite microspheres induced apoptosis of MC3T3-E1 cells by lysosomal and mitochondrial pathways. Sci China Life Sci, 2018, 61: $464-475$ 
20 Liu C P, Wu T H, Lin Y L, et al. Tailoring enzyme-like activities of gold nanoclusters by polymeric tertiary amines for protecting neurons against oxidative stress. Small, 2016, 12: 4127-4135

21 Hyslop P A, Zhang Z, Pearson D V, et al. Measurement of striatal $\mathrm{H}_{2} \mathrm{O}_{2}$ by microdialysis following global forebrain ischemia and reperfusion in the rat: Correlation with the cytotoxic potential of $\mathrm{H}_{2} \mathrm{O}_{2}$ in vitro. Brain Res, 1995, 671: 181-186

22 Fan W, Qi Y, Wang R, et al. Calcium carbonate-methylene blue na- nohybrids for photodynamic therapy and ultrasound imaging. Sci China Life Sci, 2018, 61: 483-491

23 Liu C P, Wu T H, Liu C Y, et al. Self-supplying $\mathrm{O}_{2}$ through the catalase-like activity of gold nanoclusters for photodynamic therapy against hypoxic cancer cells. Small, 2017, 13: 1700278

24 Hameed S, Bhattarai P, Dai Z. Nanotherapeutic approaches targeting angiogenesis and immune dysfunction in tumor microenvironment. Sci China Life Sci, 2018, 61: 380-391 